Cahiers $d u$ MONDE RUSSE

\section{Cahiers du monde russe}

Russie - Empire russe - Union soviétique et États indépendants

$50 / 1 \mid 2009$

Écrits personnels. Russie XVIII ${ }^{\mathrm{e}} \mathrm{XX} \mathrm{X}^{\mathrm{e}}$ siècles

\title{
L'injonction autobiographique dans les années
}

\section{0}

G.A. Medynskij et l'histoire du métro de Moscou

Josette BOUVARD

\section{(2) OpenEdition}

Journals

Édition électronique

URL : https://journals.openedition.org/monderusse/9154

DOI : 10.4000/monderusse.9154

ISSN : $1777-5388$

Éditeur

Éditions de l'EHESS

Édition imprimée

Date de publication : 31 mars 2009

Pagination : 69-92

ISBN : 978-2-7132-2259-7

ISSN : $1252-6576$

Référence électronique

Josette BOUVARD, "L'injonction autobiographique dans les années 1930 », Cahiers du monde russe [En ligne], 50/1 | 2009, mis en ligne le 01 janvier 2009, consulté le 02 septembre 2022. URL : http:// journals.openedition.org/monderusse/9154; DOI : https://doi.org/10.4000/monderusse.9154 


\title{
L'INJONCTION AUTOBIOGRAPHIQUE DANS LES ANNÉES 1930
}

\author{
G.A. Medynskij et l'histoire du métro de Moscou
}

Le projet de Grigorij Medynskij d'impulser le mouvement des journaux ouvriers du métro de Moscou en 1934 s'insère dans le contexte plus général de l'entreprise gorkienne d'écriture de l'histoire des fabriques et des usines et soulève les problèmes de la subjectivité et de la construction de soi pendant la période stalinienne. L'étude de la subjectivité des acteurs a intégré le champ de l'histoire sociale russo-soviétique dans le cadre de la microhistoire depuis plusieurs années déjà ${ }^{1}$ mais cette approche, en ce qui concerne le projet de Gor'kij, suscite encore de multiples réticences. On doit à Jochen Hellbeck ${ }^{2}$ d'avoir ouvert la voie avec l'étude du journal de Stepan Podlubnyj (1931-1939) dans laquelle il analyse l'usage du journal personnel comme construction de l'identité du diariste en fonction des normes sociales et des outils disponibles de définition de soi. Cette approche se justifie d'autant plus pour l'étude des journaux personnels, suscités et dirigés, de l'histoire des usines.

\section{Les annales de la construction socialiste}

Le projet d'écriture de l'histoire des fabriques et des usines, soutenu par le Politburo ${ }^{3}$ et mis en œuvre pendant les années 1931-1936, doit tout à son initiateur Maksim

1. Voir notamment Sheila Fitzpatrick, éd., Stalinism New Directions, Londres-New York : Routledge, 2000 ; Stephen Kotkin Magnetic Mountain : Stalinism as Civilization, Berkeley : University of California Press, 1995

2. Jochen Hellbeck, « Fashioning the Stalinist Soul : The Diary of Stepan Podlubnyj (19311939) », in Fitzpatrick., éd., Stalinism New Directions, p. 77-118 et Revolution on My Mind : Writing a Diary under Stalin, Londres : Harvard University Press, 2006.

3. RGASPI (Rossijskij gosudarstvennyj arhiv social'no-političeskoj istorii - Archives nationales russes d'histoire socio-politique), f. 17, op. 3, d.853. Résolution du Politburo du 
Gor'kij. L'Histoire des usines ${ }^{4}$ appartient aux grands projets discursifs de Gor'kij qu'il concevait comme de vastes «encyclopédies », apologies de la construction socialiste : «Histoire de la guerre civile» (en partie réalisée), «Histoire des campagnes », « Histoire des villes », « Histoire de la culture et du mode vie », « Histoire des femmes » (projets non réalisés). Le paradigme du bilan « historique » résume la conception de Gor'kij. Ce projet de propagande scripturaire est fondé sur la révision du passé en fonction du présent et repose sur le principe d'écriture par les acteurs eux-mêmes, accompagnés dans leurs tâches par des «spécialistes », historiens et écrivains professionnels. Dans l'esprit de Gor'kij, le projet possède une visée éducative et culturelle : former de « véritables prolétaires » «conscients » de leur travail et capables de procéder à une « perestroïka » de leur personnalité. Les thèmes de la prise de conscience, du bilan du travail accompli et de la perfection de soi sont récurrents dans les textes injonctifs comme dans la plupart des journaux étudiés. En ce sens, cette étude s'inscrit dans la lignée du travail de Jochen Hellbeck, mais son

10 octobre 1931, date officielle de la création de l'entreprise, publiée dans la Pravda ${ }^{\circ} 281$ du 11 octobre 1931. La résolution fut préparée par le responsable de l'Agitprop du CC du Pc(b) A.I. Stetskij. À l'origine trois dates : l'appel du 3 septembre 1931, lancé par Gor'kij au plénum de la RAPP (Rossijskaja associacija proletarskih pisatelej - Association russe des écrivains prolétariens), suivi de son article du 7 septembre 1931 sur l'histoire des fabriques et des usines publié dans la Pravda et les Izvestija. La résolution du Politburo qui donne son aval décide « d'accepter la proposition de Gor'kij et de commencer l'édition de séries de recueils, Histoire des usines », et précise « Ces recueils doivent donner une image des usines, de leur développement pour les usines anciennes et de leur création pour les nouvelles, décrire leur rôle dans l'économie du pays, la situation des travailleurs avant la révolution [...] la création des organisations révolutionnaires, le rôle de chaque usine dans le mouvement révolutionnaire, [...] le changement des relations à l'intérieur de l'usine après la révolution, les transformations des travailleurs, le travail de choc, l'émulation socialiste et la croissance de la production, ces dernières années ».

4. Une image très significative est donnée moins par le nombre de livres publiés que par la masse impressionnante de documents d'archives rassemblés par la rédaction centrale de l'Histoire des usines. Dans l'ensemble, 94 fabriques, usines et grands chantiers ont participé à l'entreprise de Gor'kij dont 24 de Moscou et 20 de Leningrad. Les livres publiés sous sa direction aux Éditions de l'Histoire des fabriques et des usines, OGIZ, ne dépassèrent pas la quinzaine. Il s'agit pour l'essentiel de monographies d'usines Putilov v trëh revoljucijah [Putilov à travers trois révolutions] de M. Mitelman, B. Glebov, A. Uljanskij, 1933 ; Zavod imeni Lenina, 1857-1918 [L'Usine Lenin] de l'auteur ouvrier N. Pajalin, M. : Socekgiz, 1933 ; Krasnyj Triugol'nik [Le Triangle rouge] de B. Šabalin, 1935 ; Istorija Ižorskovo zavoda [L'Histoire de 1'Usine Ižorskij] de S.Zavjalov, 1934 ; Byt rabočih trehgornoj fabriki [Le mode de vie des travailleurs de la fabrique Trehgornaja] de S. Lapickaja, 1935 ; Istorija moskovskogo intrumental'nogo zavoda [L'Histoire de l'usine d'instruments de Moscou] 1934 ; de recueils de souvenirs de travailleurs ou de collectifs : Šesnadcat' zavodov [Seize usines] M. : izd. istorija zavodov »1933 ; Istorija Belomorskogo Kanala [Histoire du canal Belomor], 1934 ; Ljudy Stalingradskogo traktornogo zavoda [Gens de l'usine de tracteurs de Stalingrad], 1934 ; Byli vysokoj gory [Légendes de la Montagne haute], 1935 ; Istorija metro Moskvy [Histoire du métro de Moscou] enfin, qui parut sous la forme de deux recueils : Rasskazy stroitelej [Récits des bâtisseurs] et Kak my stroili metro [Comment nous avons construit le métro], 1935 ; Volžskie Tkači [Les tisserands de la Volga], 1935 ; Peterburg-Moskva, istorija oktjabrskoj železnoj dorogi [Saint-PetersbourgMoscou, Histoire des chemins de fer d'Octobre], 1934, Bolševskaja trudovaja komuna [la commune de travail de Bolševo], 1936; une autobiographie Moja žizn' [Ma vie] de A. Korevanova, 1936) ; une grande partie des sources relatives au travail d'organisation de la rédaction centrale furent détruites à la fin des années 1930, voir Žuravlev, Fenomen «Istorija fabrik i zavodov», predprijatie Gor'kogo v kontekste tricatyh godov [Le phénomène de l'histoire des fabriques et des usines, l'entreprise de Gor'kij, dans le contexte des années 1930.] M. : Rossijskaja akademija Nauk, Intitut Rossijskoj Istorii, 1997,p. 13-14. 
objet se distingue considérablement des journaux d'ouvriers par son ampleur comme par sa nature. En effet, les journaux ouvriers des grandes entreprises staliniennes des années 1930 sont centrés sur le thème du travail dans l'entreprise ou le grand chantier et s'insèrent dans le cadre institutionnel de l'Histoire des fabriques et des usines. Une histoire dont l'approche scientifique et littéraire relevait de la théorie du marxisme-léninisme, d'une histoire bolchevique des usines.

Il faut toutefois rappeler que le projet de Gor'kij, s'il était soutenu par le Politburo éveillait aussi sa méfiance: l'idée de résurgence d'une culture prolétarienne née au début du siècle était contestée dans les plus hautes sphères ${ }^{5}$. Cependant, Maksim Gor'kij militait en faveur de la croissance industrielle du pays, qu'il considérait comme un moyen de sortir la Russie de "l'état d'arriération » paysanne traditionnelle, motif exploité dans la propagande stalinienne. Dans ce contexte, l'écriture biographique du journal ouvrier était valorisée comme instrument de cette prise de conscience et construction de l'Homme nouveau, thème récurrent des années $1930^{6}$.

Le développement des journaux ouvriers de l'Histoire des usines dans le court terme (1931-1936)7 s'inscrit à la charnière des années 1920 et 1930 précisément dans un moment de crise identitaire, alors que la plupart des travailleurs d'origines ethniques et sociologiques diverses sont censés se fondre dans un collectif d'avantgarde. Ces travailleurs ne répondent pas à l'image que le pouvoir bolchevik se fait de la classe ouvrière. Il est, par conséquent, impératif de les transformer en ouvriers d'usine, en prolétaires conscients. Dès lors, un vaste système de propagande se met en place à travers l'iconographie, la photographie et la presse d'entreprise qui exaltent le travailleur et la travailleuse de choc. Ce phénomène est la conséquence de la radicalisation de la politique stalinienne et du Grand Tournant, décidé par Stalin en 1928-19298. En effet, la collectivisation des campagnes provoque des mouvements

5. « Nado nažat' na CK » [Il faut faire pression sur le Comité central], chapitre du livre de Žuravlev, qui cite la lettre de Gor'kij à Tihonov le 1er février 1932, Le phénomène de l'histoire des fabriques..., p. 53. Kaganovič, lui-même membre de la rédaction centrale et curateur de l'Histoire du métro, n'affirmait-t-il pas que «l'essentiel est de construire le métro, l'histoire viendra bien après »?

6. S. Fitzpatrick, Le stalinisme au quotidien : La Russie soviétique dans les années 30, P.:

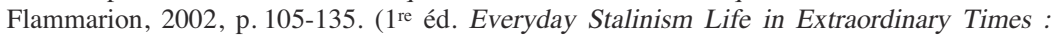
Soviet Russia in the 1930's, New York - Oxford : Oxford University Press, 1999).

7. Le projet est liquidé en 1938 (Žuravlev, Fenomen «Istorija fabrik i zavodov », p. 73-75). Les dirigeants M.P. Tomskij, S.G. Bron de la maison d'édition GIIST (ex-OGIZ) furent arrêtés. Tomskij se suicida en 1936. Leur successeur P.F. Judin déclara que la rédaction était une réserve d'éléments trotsko-boukhariniens. Pendant toute l'année 1937, ce fut toute la vieille garde gorkienne qui fut exclue de l'Histoire des usines : N. Šučkanov, A. Gajsinovič, E. Grekulov, et bien d'autres. La rédaction cessa son activité à la fin de 1937. Les livres à paraître furent mis au pilon, les archives liquidées, certains documents, parmi lesquels des livres de Gor'kij lui-même, furent détruits, classés caducs par le décret du 31 janvier 1938. À la rédaction de l'Histoire du métro, le secrétaire de rédaction Kosarev et Averbah furent arrêtés et fusillés et Rezničenko déporté.

8. L'expression utilisée par Stalin dans son article paru dans la Pravda du 7 novembre 1929 $\mathrm{n}^{\circ} 259$ désigne la période de rupture avec la NEP instaurée par Lenin et la politique conduite pendant le Premier Plan quinquennal de radicalisation économique, « liquidation des koulaks en tant que classe », collectivisation des campagnes, industrialisation à marche forcée 1929- 
migratoires de grande ampleur influant sur la structure sociale des villes qui se ruralisent. On observe une mutation sociologique des ouvriers d'usines, pour beaucoup des paysans récemment urbanisés, des saisonniers ${ }^{9}$ qu'il faut éduquer, transformer en ouvriers capables de mener à bien leur tâche productive. Par ailleurs, le tournant industriel du Premier Plan quinquennal soulève les problèmes récurrents de la fluidité des travailleurs, de la productivité, de la discipline du travail, de la réalisation du plan de production, de la motivation des travailleurs et de la maîtrise de la technique ${ }^{10}$. Cette radicalisation s'accompagne d'un mouvement de contestation des hiérarchies et des générations précédentes, accusées de lenteur, de sabotage, d'inefficacité bureaucratique. Ce sont les jeunes komsomols qui en constituent le fer de lance et ce sont eux, en majorité, qui rédigeront leurs journaux. Cet ensemble de questions et d'impératifs idéologiques trouve un écho dans la théorie du journal ouvrier.

Il convient de s'interroger sur les procédures de légitimation et de validation de soi qui ont permis aux travailleurs de s'intégrer. Si les mesures disciplinaires et répressives ont opéré une fonction essentielle dans la pénalisation du travail, la lutte contre l'absentéisme ${ }^{11}$, l'exclusion «d'éléments socialement étrangers »12, elles se sont combinées à d'autres formes incitatives du travail ${ }^{13}$. La promotion d'une élite ouvrière - les travailleurs de choc puis, à partir de 1936, les stakhanovistes - est un élément de cette politique. L'histoire des grands complexes industriels créés dans les années 1930 met en évidence les pratiques sociales instituées pour fixer les travailleurs à leurs postes, réduire les rotations de personnel, augmenter la productivité, construire une identité ouvrière, pivot du système ${ }^{14}$. La mise en voix de cette identité « prolétarienne » - identité toujours à redéfinir en tant que discours tenu sur

1931. Commencée en 1928 par une crise des collectes dans les campagnes, la politique stalinienne se radicalise en 1929. En juin 1929, Stalin décide d'une nouvelle phase de développement avec « la collectivisation de masse », cf. Nicolas Werth, «Un État contre son peuple : violences, répressions, terreur en Union soviétique » in, S. Courtois, N. Werth, J.-L. Panné, Le livre noir du communisme : Crimes, terreur et répression, P. : Robert Laffont, 1997, p. 49-289.

9. Moshe Lewin, La formation du système soviétique : essai sur l'histoire sociale de la Russie dans l'entre-deux-guerres, P.: Gallimard (Bibliothèque des Histoires), 1987 ; David Hoffmann, Peasant Metropolis : Social Identities in Moscow, 1929-1941, Ithaca : Cornell University Press, 1994.

10. Jean-Paul Depretto, Les ouvriers en URSS, 1928-1941, P. : Publications de la Sorbonne, Institut d'études slaves, 1997.

11. Lewin, La Formation du système soviétique et Le siècle soviétique, P. : Fayard, 2003.

12. Werth, «Un État contre son peuple... » ; Nathalie Moine, « Le pouvoir bolchevik face au petit peuple urbain. Clivages sociaux, assignations des identités et acculturation à Moscou dans les années 1930 », thèse, Lyon II, janvier 2000.

13. Fitzpatrick, Everyday Stalinism Life ; Kotkin, Magnetic Mountain.

14. Lewis H. Siegelbaum, Ronald G. Suny, éds., Making Workers Soviet : Power, Class and Identity, Ithaca - Londres : Cornell University Press, 1994 ; Donald A. Filtzer, Soviet Workers and Stalinist Industrialisation: The Formation of Modern Soviet Production Relations, Armonk, NY, 1986 ; Sergej V. Žuravlev, « Malen’kie ljudi » $i$ «bo'lšaja istorija » : Inostrancy moskovskogo elektrozavoda v sovetskom obščestve, 1920h-1930h gg. [ "Les petites gens » et « la grande histoire»: les étrangers de la centrale électrique de Moscou dans la société soviétique, 1920-1930], M. : Rosspen, 2000. 
le prolétaire - est au cœur du projet de Gor'kij et de son disciple, G.A. Medynskij. L'écriture du travail et sa méthodologie ont une fonction pragmatique et économique d'augmentation de la performance et de la productivité. En ce sens, c'est une forme soviétique de ce qui sera dénommé coaching ${ }^{15}$ dans l'entreprise moderne.

L'objet de cet article se restreindra à l'analyse des techniques de soi qui relèvent de la pédagogie du journal ouvrier liée à la personnalité du pédagogue et propagandiste que fut Grigorij Medynskij.

\section{À l'usine comme à l'école}

Peut-on apprendre à tenir son journal ? Au sein de l'institution politique, historique et littéraire de l'Histoire des usines, un apprentissage collectif du journal, par essence singulier, est-il possible ? La caractéristique du journal ouvrier, suscité, encadré et contrôlé n'entraîne-t-elle pas une série de difficultés tant pour les scripteurs que pour leur instigateur ? Le journal personnel peut-il être transformé en un exercice de style conjugué à toutes les personnes du singulier, censées fusionner dans une troisième personne du pluriel, celle de l'écriture bolchevique de l'histoire, qui plus est précisément celle de l'année 1934 ?

C'est à ce phénomène que fut confronté Medynskij dans sa pratique pédagogique et ses textes théoriques de direction du journal ouvrier au sein de la rédaction de l'Histoire du métro de Moscou (1934-1935). L’injonction autobiographique présupposait la pratique régulière de l'écriture par les ouvriers du métro sur les lieux de travail, pour le métro au fond des puits, et le soir après des journées harassantes.

Si la fonction injonctive consiste à orienter le comportement et la conduite du récepteur, les textes de Medynskij relèvent bien d'un type de discours de propagande. Ils ne constituent que l'un des éléments d'un vaste dispositif institutionnel de textes - décrets, résolutions, ordres - visant à encadrer, réglementer, contrôler le comportement du citoyen soviétique et, a fortiori, celui de l'ouvrier d'usine ; ils apparaissent comme autant d'actes de parole qui, sous le stalinisme, ont imposé une norme, voire entraîné l'exclusion de la vie publique. Le lexique de l'injonction nado, nužno (kak nado pisat'), Napišem [il faut, il est nécessaire d'écrire (comment doit-on écrire), Écrivons] - est récurrent dans les slogans et les articles des années 1930, en particulier dans les tracts, les instructions et l'aide méthodique de l'Histoire des usines, de sorte que l'on peut lire ces journaux ouvriers et le discours pédagogique d'encadrement comme autant de variations discursives sur le mode personnel $\mathrm{du}$ système plus global de la contrainte du régime stalinien. Il s'agit alors d'évaluer le degré d'intériorisation par les acteurs ainsi que leur participation héroïque et collective comme une contribution à la tâche de mobilisation et de propagande. Or les auteurs ouvriers ne disposaient pas d'autres outils ni de système de valeurs pour se situer dans le contexte historique et idéologique qui était le leur. Seuls les anciens

15. Eric Pezet, éd., Management et conduite de soi : Enquête sur les ascèses de la perfomance, P. : Vuibert, 2007. 
ouvriers pouvaient témoigner d'une expérience ouvrière, syndicale et révolutionnaire différente. On ne fit appel à eux qu'au début du projet et dans un cadre de parole contrôlé. Par ailleurs, l'injonction autobiographique ne présume pas l'acceptation par les intéressés du rôle qui leur est assigné. Ils peuvent s'y soustraire, le contourner, l'interpréter, en assumer une vision plus personnelle (déformée, selon les responsables) ou encore, reprendre à la lettre les consignes du pédagogue. La subjectivité, l'implication personnelle, loin d'être négligées pendant les années 1930 en Russie, apparaissent comme des pièces maîtresses du régime. Les «dobrovolcy»engagés volontaires du journal ouvrier - constituent, à cette époque cruciale de l'histoire soviétique, une expérience unique dans l'histoire du journal ${ }^{16}$.

Le principal initiateur et «directeur de conscience », Grigorij Aleksandrovič Medynskij (Pokrovskij), 1899-1984, écrivain «prolétarien» et pédagogue de formation avait les qualités requises. Propulsé par Averbah, alors secrétaire de la rédaction centrale de l'Histoire des usines, il s'implique à la direction de l'Histoire du métro de janvier à décembre 1934, dans ce mouvement de masse des journaux ouvriers (massovoe dviženie vedenija dnevnikov) qui ne fut d'ailleurs pas un mouvement et encore moins un mouvement de masse. Reconnaissons à Medynskij d'en avoir pris conscience très tôt dans sa lettre adressée fin 1934 à la direction de Literaturnaja Gazeta :

Cent auteurs ouvriers, c'est 40000 courriers Hlestakov, ni plus ni moins. Quiconque a écrit ses souvenirs, ou a commencé un journal et l'a arrêté, ne peut être appelé auteur ouvrier. Ce titre est exigeant et oblige à un travail sur soimême et sur la langue. Et de tels auteurs sont en quantité négligeable au métro. ${ }^{17}$

Medynskij impulse l'écriture, il s'investit dans la didactique du journal, prodigue des conseils aux auteurs ouvriers. Plus encore, il opère la synthèse de la pratique diaristique dans un ensemble cohérent de textes et de discours ${ }^{18}$. Il se consacre à l'injonction autobiographique au sens large du terme. Or, il n'y a pas d'injonction

16. Peu de journaux sont archivés. Si les listes d'auteurs oscillent entre 150 et 200 auteurs, seuls 36 sont recensés par ordre alphabétique, GARF (Gosudarstvennyj arhiv Rossijskoj Federacii - Archives d'État de la Fédération de Russie), f. 7952, op. 7, Istorija fabrik $i$ zavodov [Histoire des fabriques et des usines], d. 447-457. Ce phénomène s'explique par le fait que tous les auteurs n'ont pas rendu leurs manuscrits à la rédaction ou les ont détruits. Certains ont commencé quelques entrées puis ont abandonné, faute de compétence, de temps et de disponibilité. En aucun cas on ne peut estimer qu'il s'agit «d'un mouvement de masse ». Rappellons qu'en 1934-1935 plus de 75000 ouvriers travaillaient à la construction du métro.

17. GARF, f. 7952, op. 7, d. 142 .

18. Ces documents sont conservés aux archives du GARF, de 1'IMLI (Institut mirovoj literatury im. A.M. Gor'kogo - Institut de littérature mondiale A.M. Gor'kij) qui détient toutes les archives de Gor'kij, et du RGALI (Rossijskij gosudarstvennyj arhiv literatury i iskusstva Archives russes d'État de littérature et d'art). 405 dossiers du fonds 7952, op. 7 du GARF sont consacrés aux divers aspects de l'histoire du métro de Moscou. On y trouve notamment une masse considérable de documents rassemblés par la rédaction de l'Histoire du métro, chargée au début de 1934 d'écrire l'histoire de la construction et qui a publié deux livres en mars et mai 1935, Rasskazy stroitelej et Kak my stroili metro. Les matériaux de la rédaction (1934-1935) se trouvent dans les dossiers 219 à 496, les journaux et œuvres littéraires des travailleurs du métro dans les dossiers 447 à 460 . 
sans interaction et Medynskij poursuit un dialogue actif avec les auteurs ouvriers qu'il prend en charge. C'est la première caractéristique de son travail à la rédaction de l'Histoire du métro. La seconde, la plus importante, Medynskij s'applique à construire une réflexion générique sur le journal personnel au sein de l'institution de l'Histoire des fabriques et des usines.

La production discursive de Medynskij présente ainsi un objet qui dépasse son activité éphémère à la rédaction. D'une part, ses textes concentrent toutes les annotations et réflexions métalinguistiques sur la pratique même du journal, disséminées dans les journaux ouvriers mais aussi dans les journaux personnels. D'autre part, Medynskij procède à une véritable systématisation et met en évidence la fonction centrale du journal dans la littérature ; il contextualise cette pratique dans un ensemble plus vaste, la littérature et la culture soviétiques des années 1930. S’il n'est pas le seul à le dire - c'est le discours des écrivains soviétiques au congrès de l'Union des écrivains d'août 1934 - il est l'un des rares à suivre Gor'kij à la lettre, à se frotter aux textes parfois rugueux de l'écriture ouvrière et à admettre, bien des années plus tard, l'échec de cette belle idée ${ }^{19}$.

L'activité de Medynskij peut être appréhendée sous son aspect personnel et l'on peut s'interroger sur les éléments de sa biographie qui lui confèrent autorité et le légitiment dans sa fonction d'animateur.

Un bref détour par sa biographie s'impose donc, l'analyse abordera ensuite le type de discours théorique sur le journal ${ }^{20}$ qu'il produisit à partir de sa pratique pédagogique. Medynskij réfléchit sur son travail d'écrivain tout au long de sa vie, élaborant à chaque étape un bilan de son activité passée. On peut ainsi distinguer trois strates de textes et étapes historiques distinctes : les années 1920 et 1930, années de son activité antireligieuse puis « prolétarienne », le bilan qu'il en déduit en 1957 dans une note biographique pendant la période krouchtchévienne ${ }^{21}$, enfin le texte de ses Mémoires, Stupeni Žizni, écrit à la fin de sa vie, de 1974 à 1979, pendant la période brejnévienne et publié en 1981. Il considéra l'œuvre publiée, moins comme l'histoire de sa propre vie, une véritable autobiographie que, comme le rappelle le sous-titre, «un récit lyrico-journalistique». Dans cette œuvre, Medynskij affirme avoir utilisé très largement ses archives personnelles, le journal intime des premières années (introuvable malheureusement), ses poèmes prolétariens, sa correspondance, ses textes de conférences, les lettres de lecteurs, des extraits de journaux de l'époque. Cette « biographie spirituelle », terme utilisé par Medynskij lui-même, souffre d'un jugement rétrospectif inhérent à ce type de récit.

Les sources le concernant sont réparties dans trois centres d'archives moscovites : les archives Gor'kij de l'Institut de littérature mondiale pour les documents du Secrétariat de l'histoire des usines (IMLI), le fonds de l'Histoire des

19. Stupeni žizni [Les degrés de la vie], M. : Sovetskij Pisatel`1981, p. 146 : « Je donnai mon accord, et dirai-je, fonçai, ce que par la suite je regrettai beaucoup tant cette histoire fut contraignante et stérile. » Deuxième édition posthume, M., 1985.

20. Dnevnik signifie « écrit au jour le jour » (de den’, le jour) ; il désigne aussi bien le journal intime ou personnel que l'agenda.

21. RGALI, f. 20504, op. 1, d. 105,1. 1-5, daté du 5 août 1957. 
Fabriques et des Usines du GARF22 pour tous les documents de la rédaction de l'histoire du métro (sténogrammes de réunions de la rédaction, manuscrits des auteurs ouvriers). Enfin le RGALI conserve le fonds personnel, une partie de la correspondance de Medynskij, encore fermé aux chercheurs car l'auteur est mort en 1984. Ce qui reste accessible se trouve dans le fonds du secrétariat de la Direction de l'Union des écrivains ${ }^{23}$, en particulier cinq pages d'une autobiographie consistant en un exemplaire dactylographié, corrigé par Medynskij, daté d'août 195724, destiné au secrétariat de l'Union des écrivains, document interne à l'institution. Medynskij réécrit constamment l'histoire des années 1930, essayant d'expliquer ce qu'il fut et s'efforçant de maintenir la cohérence d'une vie commencée à dix-sept ans à la sortie du lycée en 1917 en pleine révolution de Février pour s'achever à l'ère brejnévienne. Il a donc vécu « de fait 40 ans de [sa] vie consciente sous le pouvoir soviétique et sans autres intérêts que ceux du pouvoir soviétique ${ }^{25}$. Le cadre de l'autojustification de sa vie et ses valeurs référentielles ne peuvent se penser en dehors du système soviétique. Son regard critique, voire ironique, sur son activisme passé s'insère dans ce contexte biographique.

\section{Un intellectuel soviétique ordinaire}

La lecture des textes écrits comme de ses interventions orales révèle l'image d'un locuteur totalement impliqué dans son discours. Mais qui est réellement Medynskij26 ? Écrivain et pédagogue, il a 35 ans en 1934, a publié plusieurs nouvelles et un roman lorsqu'il assume la charge du journal ouvrier à la direction de l'Histoire du métro. Il se revendique dans ses pages autobiographiques rédigées en 195727 comme un homme ordinaire et affirme : « je dirai sincèrement qu'il ne m'est pas très facile de parler de moi » (biffure « écrire son autobiographie »). Il constate dans ce texte qu'il n'a pas pris part à la guerre ni accompli d'actes remarquables susceptibles d'intéresser un lecteur, qu'il n'est pas un « héros ». Il se définit comme un homme du siècle passé (il est né en 1899) qui, par ses origines - il est le fils « d'un pope de campagne » - appartient « au vieux monde ». Son parcours biographique relate le chemin qui l'a conduit « de ses origines paysannes à l'écrivain soviétique qu'il est devenu ». Né avec le siècle, il reprend un leitmotiv du récit de vie soviétique en affirmant «être né à la vie consciente avec la révolution bolchevique ». Il a cependant terminé ses études secondaires avant la révolution et n'a pas participé à

22. GARF, f. 7952, op. 7, d. 219-499.

23. RGALI, f. 631, op. 15, 20, 25, 26.

24. RGALI, f. 2504, op. 1, d. 105.

25. Ibid., 1. 1.

26. G.A. Medynskij, de son vrai nom Pokrovskij, tient son pseudonyme de la ville de Medyn' où il a commencé à travailler au Conseil de district de l'économie communale. Il a également publié sous le nom de Stoljarov.

27. RGALI, f. 2504, op. 1, d. 105, 1957, pages destinées à l’Union des écrivains. 
la guerre civile (il fut réformé par l'Armée rouge pour sa myopie). Il insiste dans son texte de 1957 sur la rupture intervenue dans la vie de ses contemporains : " mon parcours de vie est celui des gens de ma génération qui, de fait, a vécu sous le pouvoir soviétique », «Chacun, dit-il, a suivi une voie particulière au socialisme ». Il appartient à une génération qui a pensé et engagé sa vie sous le régime soviétique à une période cruciale où s'est présentée à lui la possibilité d'une carrière d'écrivain. Le passage du singulier au collectif, de l'universalisation de l'expérience personnelle que l'on voit s'opérer dans le texte de Medynskij, est récurrent dans les autobiographies de l'Histoire des usines. En ce sens, cette courte autobiographie répond aux critères biographiques qui se sont institués dans les années 1930. Medynskij analyse son engagement dans la lutte antireligieuse des années 1929-1930 par sa relation à son père qui était un pope de village et par sa volonté de rupture avec le vieux monde, avec son passé28, comme il le souligne à nouveau en 1981 : «Fils de prêtre, c'est précisément à cause de cela que je devins un athée violent, sans compromis, un "sansdieu", un combattant de dieu même et je le reste encore $»^{29}$. En 1957, il présente sa première nouvelle, $\mathrm{Med}^{30}$, « comme de la propagande typiquement antireligieuse ». Il substitue à la morale religieuse de son enfance la morale laïque du bolchevisme, au bénéfice social et culturel de la révolution. À travers ses textes, l'énonciateur construit son parcours intellectuel et personnel d'adhésion au bolchevisme.

Pédagogue, de 1919 à 1929 il est instituteur, puis instructeur. Le 7 novembre 1922, il est engagé au centre Pokrovskij ${ }^{31}$ à Moscou où, pendant sept ans, il occupera successivement les postes d'éducateur, de directeur de section, puis de directeur du centre $^{32}$ qui relève alors du ministère de l'Éducation. Véritable tragédie, conséquence de la grande famine de 1921-1922, des dizaines de milliers de jeunes vagabonds, bezprizorniki, se retrouvent livrés à eux-mêmes. Ils trouvent refuge dans le centre Pokrovskij où avec un manque criant de moyens un petit nombre de travailleurs sociaux, dont Medynskij, les prennent en charge ${ }^{33}$. Medynskij participe à un «laboratoire psychologique » créé par l'équipe éducative. Avec d'autres pédagogues, il accumule des centaines d'entretiens avec les vagabonds ${ }^{34}$. Les éducateurs établissent pour chacun d'entre eux une fiche individuelle, harakteristika, puis le conseil pédagogique les oriente vers différents orphelinats. Parmi eux se trouvent de jeunes voleurs, des délinquants auprès desquels les pédagogues mènent un difficile

28. Ibid.,1. 2

29. Stupeni žizni, p. 7.

30. Med [Le Miel], M. : Molodaja Gvardija, 1925.

31. Alors situé au 81, rue Bakunin à Moscou, dans un ancien monastère de femmes, ibid., p. 118.

32. Ibid., chap. V, « Pokrovskij priemnik », p. 114-132.

33. Ibid. p. 113. Les orphelinats prévus pour une cinquantaine d'enfants durent en héberger de 150 à 200 dans des conditions déplorables.

34. Certains de ces entretiens ont été conservés par l'auteur et sont cités (ibid. p. 124-125). Medynskij, qui, en 1929, mène parallèlement le combat antireligieux, s'intéresse particulièrement à leur représentation de la religion. 
travail de réinsertion dans les centres et les usines de la région. Leur objectif est la rééducation par le travail ou l'envoi dans des communes d'enfants, comme celle de Bolševo. Nommé directeur d'un centre pour enfants particulièrement difficiles dans l'ancien bâtiment du centre Rukavičnikovskij en 1927, il note un changement dans le comportement des vagabonds, plus endurcis et moins réceptifs aux impératifs de rééducation. Ces phénomènes sont ressentis par Medynskij comme un échec. Comprenant qu'il n'est pas Makarenko, il cherche à se reconvertir. Sa pratique de pédagogue le conduit à suivre les conférences de Gastev ${ }^{35}$. Il prépare l'examen de l'université d'État de la rue Usačevskaja (Institut pédagogique). Admis, il est finalement recalé en raison de ses origines sociales ${ }^{36}$. En 1928, il quitte son travail pour l'éducation de jeunes adultes ouvriers dans la banlieue de Moscou.

En 1957, il considère ses activités de jeunesse « comme les jalons extérieurs de sa biographie, l'essentiel de son développement ayant suivi la voie de ses intérêts les plus intimes, affaire de conscience ».

Parmi ses nombreuses activités, l'activisme antireligieux, comme il l'a expliqué lui-même, occupe une place importante jusqu'en 1933 : «Je voulais régler mes comptes avec la religion, le plus rapidement possible et pour ainsi dire dans un combat ouvert au corps à corps $»^{37}$. Son travail de propagandiste antireligieux est très prolifique ${ }^{38}$. En effet, de 1927 à 1929, il multiplie les conférences et rédige une série de brochures et de pamphlets antireligieux ${ }^{39}$. Il termine la série par un essai publié en $1934^{40}$, et envoie en 1936 une lettre ${ }^{41}$ désenchantée à Stavskij sur son

35. Aleksej Kapitonovič Gastev (1882-1941) est une personnalité unique de la littérature prolétarienne. Révolutionnaire de la première heure, poète prolétarien, il célèbre l'ouvrier et le thème industriel dans ses poèmes dès le début du siècle, Poezija rabočego udara [La poésie du coup ouvrier], 1918; My roždaemsja iz železa [Nous naissons du fer], Gudki [Les sirènes], Pačka orderov [Le paquet de mandats], 1921. Théoricien, il écrit entre autres sur la culture : Vosstanie kul'tury [Le soulèvement de la culture], 1923 ; Novaja kul'turnaja ustanovka [La nouvelle situation culturelle], 1923. Mais surtout, il devient l'un des rationnalisateurs du travail en organisant en 1920 l'Institut central du travail à Moscou auprès du Comité central des syndicats, VCSPS, qu'il dirigea jusqu'en 1938. Il travaille sur le geste ouvrier et élabore une nouvelle méthode de standardisation du travail, fondée sur la décomposition et l'analyse des gestes au travail. Auteur prolifique, il publie en 1927 les livres Kak nado rabotat' [Comment il faut travailler], Ustanovka proizvodstva metodom [La mise en place de la méthode de production], Rekonstrukcija proizvodstva [La reconstruction de la production], (Bolšaja sovetskaja enciklopedija, t. 14, 1929 et t. 6, 1971).

36. Stupeni Žizni, p. 124.

37. RGALI, f. 2504 op. $11.3,1957$.

38. Il évoque son activisme dans Stupeni žizni. Il participe au séminaire antireligieux, organisé au district Bauman du parti par Fedor Putincev. Cette période de sa vie est relatée avec ironie par le vieil homme des années 1980.

39. Buržuazija i religija [La bourgeoisie et la religion], M. : Ateist, 1928 ; Mučenik bogoiskatel'stva (Dostoevskij i religija) [Dostoevskij et la religion], M. : Ateist, 1929 ; Esenin, eseninščina i religija [Esenin, eseninščina et la religion], M. : Ateist, 1929; Poet Nekrasov i religija [Le poète Nekrasov et la religion], M. : Ateist, 1929.

40. Religioznye vlijanija $v$ russkoj literature [Les influences religieuses dans la littérature russe], M., 1933, sur la couverture 1934. Medynskij dans le texte de 1957 indique 1934 comme date de publication.

41. RGALI, op. 631, d. 15,1. 138, lettre du 16 novembre 1936. 
essai Pisateli i religija [Les écrivains et la religion] dont la publication passe inaperçue : Medynskij considère ce thème comme l'un des plus importants pour comprendre l'héritage littéraire. Il conserve un article non publié sur Gor'kij et la construction de Dieu. Cette phase active de propagande antireligieuse prend fin, tournant qui correspond également à une mise au pas des radicaux de l'athéisme militant. Il passe alors de la destruction de Dieu à l'édification de l'ouvrier modèle. Il se tourne vers la prose et se consacre à l'étude du monde ouvrier pendant le Premier Plan quinquennal, thème en vogue au début des années 1930. Il publie un roman en $1931^{42}$, dont l'idée principale est la construction d'une nouvelle fabrique du Plan quinquennal dans le village d'Ivanteevka : «C'est un roman sur la construction, les bâtisseurs et en même temps sur l'éducation des gens ». Pour cette œuvre, il s'engage dans la fabrique en tant que travailleur culturel, kul'trabotnik, vit dans les baraquements avec les ouvriers, organise des soirées littéraires sur les lieux. Le roman, accueilli par une critique favorable dans la presse, lui apporte une certaine renommée qui lui permet d'être admis à la MAPP ${ }^{43}$ dirigée à l'époque par V. M. Kiršon, L.L. Averbah, B. Gorbatov, V.V. Ermilov, «l'aréopage » des dirigeants de la RAPP. Il fait son entrée en littérature par la petite porte des écrivains prolétariens, lui qui, écrit-il en 1984, de par ses origines sociales, n'osait même pas y penser. Il participe à l'activisme des écrivains prolétariens avec une foi de néophyte. Lors de «l'appel des udarniki [travailleurs de choc] » à la littérature, il dirige le secteur de Hamovniki, se rend dans les usines « à la recherche de talents cachés ${ }^{44}$, puis on lui propose de diriger le «Cabinet de l'udarnik» destiné à recevoir et informer les travailleurs de choc et les auteurs ouvriers. Lorsque le 23 avril $1932^{45}$ l'activité de la MAPP est supprimée avec tous les autres groupes littéraires, Medynskij se retrouve au chômage. Averbah lui propose de travailler comme consultant littéraire à la rédaction de l'Histoire du métro nouvellement créée. Son expérience d'activiste au sein du groupe des écrivains prolétariens permet de comprendre les motivations de son engagement à la rédaction de l'Histoire $d u$ métro au début de 1934.

\section{Medynskij et l'Histoire du métro}

Medynskij est engagé officiellement à la rédaction de l'Histoire du métro le 7 février 1934 ; le 16 février, il élabore Metodičeskie ukazanija o vedenii rabočih

42. Samostroj [Autoconstruction], M. : Molodaja Gvardija, 1931.

43. Moskovskaja associacija proletarskih pisatelej - Association moscovite des écrivains prolétariens, 1925-1932.

44. Stupeni žizni, p. 144.

45. Postanovlenie CK VKP(b) o perestrojke literaturno-hudožestvennyh organizacij [Décret du Comité central du PC(b) «Sur la reconstruction des institutions littéraires et artistiques »] du 23/04/1932. Le comité d'organisation de la nouvelle Union des écrivains soviétiques commença ses réunions peu après. G. Medynskij fut membre de l'Union des écrivains (SSP Sojuz Sovetskih Pisatelej). 
dnevnikov [Aide méthodique sur la tenue de journaux ouvriers] ${ }^{46}$; la brochure est imprimée le 19 mars $^{47}$. Membre actif de la rédaction, il intervient à de nombreuses reprises aux réunions de la rédaction. Un conflit personnel l'oppose à Kulagin, secrétaire de la rédaction et membre du parti, qu'il considère comme un bureaucrate imbu de son pouvoir, violent dans ses rapports aux autres, incompétent et nuisible au projet. Celui-ci réclame à plusieurs reprises auprès de la rédaction centrale l'exclusion de Medynskij qui démissionnera de lui-même quelques mois plus tard. C'est ainsi qu'il explique les faits dans son autobiographie ${ }^{48}$.

Le 4 mars 1934, il fait un exposé sur le journal ${ }^{49}$, organise un séminaire sur le journal ouvrier ${ }^{50}$, et anime la réunion des auteurs ouvriers et des écrivains des 30-31 mars $1934^{51}$. Il opère une synthèse des journaux : «Dnevniki stroitelej kak istočnik istorii i kul'turnoe javlenie» [Les journaux des constructeurs du métro comme source historique et phénomène culturel $]^{52}$. Parallèlement à ce travail de propagandiste, Medynskij écrit une pièce de théâtre sur le métro de Moscou dont le tapuscrit daté du 15 juin 1935 se trouve aux archives du RGALI : Moskva nad nami, [Moscou au-dessus de nous], pièce en 4 actes et 18 tableaux (86 pages) ${ }^{53}$ dont les personnages appartiennent au groupe des mille premiers komsomols mobilisés au puits 5 bis. Il est également l'auteur de plusieurs essais sur l'histoire du métro, restés inédits, destinés au troisième tome qui n'a jamais vu le jour : Metro vošel $v$ faktah [Le métro est entré dans les faits], Plyvuny [Les sables mouvants] sur la première période de la construction, Rasskaz desjatnika [Récit d'un brigadiste], Beseda s tovariščem Orlovym [Entretien avec le camarade Orlov], Pervyi den' metro [Le premier jour du métro] ${ }^{54}$. Concrètement, il s'occupe de l'histoire au puits 29 où il organise des «consultations » sur le journal avec les ouvriers du métro. De son travail à la rédaction du métro, il se souvient beaucoup plus tard : « Je me suis donné à ce travail spontanément et passionnément parce que c'était vraiment

46. GARF f. 7952, op. 7, d. 231,1. 24 et ob.

47. L'aide méthodique est publiée dans la revue Istorija zavodov [Histoire des usines], (3-4), 1934, p. 149-151: V pomošč aktivu «istorija metro » [Pour aider l'actif de l' « Histoire du métro »]. Deux exemplaires sont conservés au GARF, f. 7952, op. 7, d. 416 et 446.

48. Stupeni žizni, p. 152-153.

49. GARF, f. 7952, op. 7, d. 248 .

50. IMLI, Archives Gor'kij, KG izd. 27-56-1, cité dans Žuravlev, Fenomen istorii fabrik. GARF, f. 7952, op. 7 d. 446, 1. 5-6. Programme d'étude du séminaire :1. La prise de parti des journaux ; 2. Formes de la lutte de classe au métro ; 3 . Du portrait littéraire dans le journal ; 4. La littérature de mémoire comme source de l'Histoire « Belles Lettres »; 5. Le sujet en littérature et la description des événements dans le journal ; 6 . Le dialogue et son utilisation dans le journal ; 7. Nouvelles formes de travail de choc dans le journal ; 8. Utilisation de la forme littéraire dans le journal ; 9. Le journal (agenda) et le journal.

51. Ibid., d. 239, 1. 132-150.

52. Paru dans Istorija fabrik i zavodov, Sbornik 3-4 (11-12), Istorija zavodov, M. : Ogiz, 1934, p. $128-140$.

53. RGALI, f. 619, op. 1, d. 1314, fonds du journal Oktjabr'. Moskva nad nami [Moscou audessus de nous], M., 1935.

54. GARF, f. 7952, op. 7 ,d. 415, 416. 
grandiose et que cela m'a enthousiasmé. J'ai compris mon travail d'abeille ouvrière récoltant le nectar de la vie fleurissant autour de moi $»^{55}$. Il reste marqué par ses amitiés nouées au métro, comme avec l'ingénieur Schmidt ${ }^{56}$. Mais c'est aussi en qualité d'écrivain qu'il intervient, considérant la réalité sociale qui l'entoure comme un matériau dans l'élaboration de son œuvre.

La pédagogie du journal ouvrier telle qu'il la conçoit repose sur un système d'évaluation, de lecture critique publique, d'autocritique du scripteur, de suggestions et d'autocorrections. Cette pratique du journal abolit les frontières traditionnelles entre la sphère privée de l'écriture et la sphère publique, celle des organisations sociales, parti et Komsomol, des clubs littéraires de l'Histoire des usines. Le secret, non pas celui de la vie privée, mais celui du jugement personnel que l'on porte sur ses compagnons de travail et supérieurs hiérarchiques, s'expose dans la pratique orale de l'autocritique lors de séances dans le cadre de l'histoire des usines $^{57}$. Le diariste écrit sur des «pages de verre » et tout le système d'écriture repose sur le mythe de la transparence ${ }^{58}$.

À travers un apprentissage qui se revendique mesurable, la critique du « spécialiste » du journal conduit les sujets écrivant à mener un travail sur soi dont le présupposé est le progrès. Les diaristes peuvent être critiqués à la fois sur le signifié et le signifiant de leurs textes.

Au-delà de sa pratique de pédagogue, Medynskij interroge l'écriture du journal à travers le prisme politico-littéraire des années 1930, l'interprétation marxiste de l'histoire et le réalisme socialiste auxquels il a adhéré. Il soulève également les problèmes fondamentaux de leur écriture : qu'est-ce qui fait événement dans le journal ? Comment décrire les gens ? Quels effets rhétoriques et littéraires sont acceptables dans l'écriture diariste ? Quels rapports le journal entretient-il à la littérature en général ? Comment s'intègre-t-il dans l'histoire du métro en particulier et dans l'histoire en général ? Quelle signification, enfin, peut-on conférer à l'écriture du journal ?

\section{Stupeni Žizni, p. 147.}

56. Ibid., cité dans Dni i gody Metrostroja, p. 82-86. Sur G.A. Medynskij (1899-1984), voir Literaturnaja Enciklopedija [Encyclopédie littéraire], V.M. Friče, P.I. Lebedev-Poljanskij, et al., éds., M. : Izdat. Kommunistečeskoj Akademii Literatury, Iskusstva i Jazyka, 1934, 7 t. Kratkaja literaturnaja encyclopedija [Dictionnaire de littérature], 1967, Russkie sovetskie pisateli prozaiki, bibliografičeskij ukazatel' 'Écrivains russes soviétiques : index bibliographique], L., 1964, t. 3, p. 80 à 85. Autobiographie de 1957, Stupeni žizni, M., 1981 et 1984.

57. Voir à ce sujet Bernard Pudal et Claude Pennetier, éds., Autobiographies, Autocritiques, Aveux dans le monde communiste, P.: Belin, 2002, et Brigitte Studer, Irène Hermann et Berthold Unfried, éds., Parler de soi sous Staline : la construction identitaire dans le communisme des années trente, P. : Éditions de la Maison des sciences de l'homme, 2002.

58. Voir à ce propos Jean Starobinski, Jean-Jacques Rousseau : La transparence et l'obstacle, P. : Plon, 1958. 


\section{Une fabrique journalière : les journaux ouvriers}

L'injonction autobiographique dans l'écriture des journaux s'apparente à l'institution scolaire ${ }^{59}$ par bien des aspects méthodologiques et pratiques. Medynskij la conçoit sous la forme d'une incitation qui passe par la valorisation de l'objet, le journal ouvrier, et des sujets, les auteurs. L'écriture des journaux revêt une importance capitale : le livre sur le métro est un livre dont les masses ne sont pas l'objet de narration mais bien les sujets écrivant ${ }^{60}$. Selon le pédagogue, par son acte de scripteur, le travailleur du métro abolit les frontières entre le travail physique et le travail intellectuel ${ }^{61}$. Medynskij insiste sur l'importance du témoignage direct des acteurs et le caractère irremplaçable de leur travail d'écriture. C'est une des formes authentiques d'investigation de la réalité et un élément incontournable de l'écriture de l'Histoire ainsi qu'il le souligne devant la rédaction de l'Histoire du métro et les auteurs ouvriers :

Il faut restituer cette histoire, pas à pas, par le biais d'enquêtes, de recueil de matériaux, non pas de souvenirs écrits pour un lecteur, mais un recueil minutieux, scrupuleux de matériaux bruts. Il est tout à fait juste que, pour le futur, il est nécessaire de tenir ces journaux et rétrospectivement ne pas se limiter à des souvenirs généraux mais mener ce travail minutieux de restitution de l'histoire des puits. ${ }^{62}$

Il faut saisir le mouvement de la mutation invisible du quotidien, le présent continu de la construction socialiste, l'évolution des pratiques sociales et de leurs affects, sténographier l'instant présent :

Refléter notre époque : nous nous développons, tout le pays vit, travaille, étudie, tout le pays transforme les liens de production, les relations sociales, la conscience des gens se transforme dans les petites choses, le détail. En ce moment, il faut saisir ces détails, les fixer pour que cet enregistrement (zapis') trouve sa place plus tard. Si on ne le fait pas, dans un an, ils auront disparu. ${ }^{63}$

Medynskij s'implique personnellement par l'organisation de rencontres avec les écrivains, de séminaires consacrés au journal : s'investir dans l'histoire des usines, dans l'écriture, c'est progresser dans la vie, tel est son message. Le narrateur ouvrier laisse sa trace, «l'arôme de la vie ». Il participe à la vie littéraire, a le sentiment d'être reconnu comme un interlocuteur valable auprès des intellectuels. Toutefois, la seule injonction ne peut y suffire, l'investissement personnel des auteurs est une condition indispensable à cette pratique. Les auteurs ouvriers les plus prolixes,

59. Françoise Simonet-Tenant, «L'injonction autobiographique à l'école », L'École des Lettres, 10, 2002-2003, p. 65-79.

60. GARF, f. 7952 op. 7, d. 416,1. 13.

61. Il intervient sur le sujet devant les auteurs ouvriers (GARF, f. 7952, op. 7 d. 233), et écrit Grani [Les limites] sur le même sujet, 1936.

62. Ibid., 1. 13, Réunion de la rédaction du 28 janvier 1934.

63. Ibid. 
Bondarev, Altuhov, Tjuhtjaev, Sapožnikov, ne suivent pas les indications de Medynskij uniquement par contrainte ou parce que l'injonction émane de l'institution. À cet égard, le sténogramme de la réunion des 30-31 mars 1934 des auteurs ouvriers est révélateur. Ceux-ci interrogent Valentin Kataev sur l'écriture de son roman Vremja vpered ! [Le Temps en avant !] ; ils cherchent à savoir si l'écrivain a tenu un journal avant d'écrire son roman, comment il crée ses personnages. Visiblement les auteurs s'intéressent à la fiction littéraire et le journal est perçu comme l'apprentissage du métier (remeslo) littéraire ${ }^{64}$. Un auteur ouvrier intervient :

Beaucoup d'entre nous tiennent un journal. Comment trouver des idées et mieux tenir son journal personnel ? Beaucoup le tiennent comme des matériaux de presse.

Et Kataev de répondre :

Ce qui est intéressant n'est pas la quantité d'heures de travail ni le salaire gagné, ce qui est intéressant, c'est ce que chaque homme pense. Tous se développent, personne ne reste sur place; si tu prends un homme maintenant et que tu le revois dans trois ans, tu verras qu'il a changé et si tu compares cet homme passé à son présent, tu compares la terre au ciel.

Le diariste reçoit des réponses à ses questions, accède à un milieu intellectuel dont les travailleurs étaient traditionnellement exclus. Cette forme de reconnaissance personnelle est à l'origine du désir d'écrire. Certains décident de commencer leur journal après la réunion. Cette reconnaissance se double de publication dans la presse locale. Le bon diariste est publié dans la presse d'entreprise Udarnik metrostroj, sur les journaux muraux dans les puits. Il est cité en exemple dans les réunions publiques, reçoit une célébrité passagère par l'intermédiaire de Medynskij. Il sera perçu socialement comme écrivant ${ }^{65}$. Medynskij valorise ses « bons élèves », il cite des extraits du journal de Bondarev, en notant le progrès accompli dans l'écriture. Il fait l'éloge du journal de Durasova pour les détails précis de sa vie quotidienne et la justesse de ton ${ }^{66}$. Medyndskij distribue l'éloge et le blâme, tout en conseillant à ses disciples d'accepter la critique en mettant leur amour-propre de côté : il n'a pas oublié les critiques qui ont accompagné ses propres débuts littéraires.

\section{Conseils pratiques}

Son enseignement repose sur une analyse des cahiers qui lui sont remis, suivie d'un travail individuel avec chaque auteur. Medynskij relève un certain nombre de

64. Voir Hellbeck, «Fashioning the Stalinist Soul : The Diary of Stepan Podlubnyj (19311939) », in Fitzpatrick., éd., Stalinism New Directions, et Revolution on My Mind.

65. GARF, f. 7952, op. 7, d. 416,1. 37.

66. Ibid., 1. 49-50. 
défauts qu'il illustre par des extraits de journaux. Ses remarques sont étayées par une lecture préalable attentive des journaux qu'il annote au dos. On trouve ainsi aux archives deux pages à l'encre bleue d'évaluation du journal de Bondarev67 :

Le journal est bon. Mais, il faut qu'il soit meilleur, sache-le. Voilà longtemps que tu tiens ton journal. De nombreux faits et des événements se sont passés et l'on ne sent pas les individus. Il est temps de les montrer; tu évoques très souvent Babenko. Mais qui est-il, comment est-il ? Parfois le flux complet des événements quotidiens peut être abandonné et retenu pour esquisser des figures particulières, les extraire du milieu commun et les décrire. Alors, par la suite, leur conduite, leur rôle seront plus clairs. Pour cela, il faut saisir les figures indispensables. La description détaillée d'individus au hasard ne peut que ralentir le cours des événements. Mais maintenant, il est temps de sentir les figures centrales du milieu que tu décris et de t'arrêter sur eux de façon plus détaillée.

En outre, il faut suivre le fil des événements et leur suite logique. Par exemple, tu prends un fait intéressant : quand le compresseur a failli s'arrêter par manque d'huile et que l'huile manquait parce qu'on n'avait pas versé d'argent. Que s'en est-il suivi ? Tu as emprunté deux réservoirs d'huile. Et après ? Qui est responsable de cela ? Quelles leçons en ont-elles été tirées ? Tu fais allusion à la dispute entre Babenko et Vovikov (que tu as présenté auparavant comme un udarnik). Tu as parlé de cailloux (et pourtant c'est de la malfaisance). Et bien quelle en est la cause ? Est-elle confirmée ? Tu as parlé du mécanicien Kušner : tu as proposé de ne pas le chasser mais de le surveiller. Et là encore, qu'en est-il advenu ? Quel travail a été fait avec Kušner ? S'est-il amendé ? Les événements ont une suite logique, ce lien, il faut le suivre. Medynskij.

Deux remarques s'imposent à propos de ces indications méthodiques. Les premières dénotent l'apprentissage du journal : d'une part, l'auteur doit maîtriser le fil chronologique du récit journalier, ne pas se perdre dans les détails de la journée de travail et, d'autre part, il doit apprendre l'art du portrait, « de la figure ». Il se conforme à l'image d'un auteur. Mais c'est également un auteur ouvrier dont les compétences textuelles s'exercent dans l'analyse du travail, des difficultés à exécuter les tâches, à passer du travail prescrit au travail réel. Ces remarques classent le journal parmi les instruments d'analyse du réel de la production socialiste dans les années 1930, un instrument pragmatique d'intégration des normes, des délais d'exécution, d'amélioration de la productivité du travail.

Les attentes du pédagogue sont multiples : il demande à l'auteur ouvrier une synthèse et une réflexion qui dépassent les simples notations. Le travail socialiste est le critère d'évaluation du journal qui repose en partie sur une classification des individus en bons et en nuisibles (vrediteli); il faut éliminer ou rééduquer les travailleurs qui freinent les « rythmes de productivité », ces impératifs apparaissent à la limite de la délation dans le contexte des années 1930. La lecture critique de Medynskij fait aussi apparaître des exigences littéraires de «personnages » qui rendent lisibles le journal et visible la réalité des puits. Medynskij insiste sur la nécessité de figures, de personnalités dans le journal. Cependant, l'auteur ouvrier 
réagit en défendant son point de vue devant le consultant. Ainsi, en haut de page, Bondarev répond :

Je suis reconnaissant au camarade Medynskij pour ses conseils, mais je pense que je n'ai pas encore de personnage central. Il y a des personnages, mais il est trop tôt pour les fixer sur les lieux. Ce serait mal de les fixer, ils n'apparaissent pas sur les lieux. Bondarev.

Les marges sont ainsi utilisées pour instaurer un espace de dialogue entre l'auteur ouvrier et le pédagogue. Lors d'une réunion publique, Medynskij aborde le journal de Bondarev ${ }^{68}$ et explique qu'en travaillant avec lui, celui-ci a corrigé son défaut, l'absence d'individus. Deux participants sont invités à lire les descriptions de Kučerenko dans les journaux de Babanov et dans celui de Bondarev ; Medynskij fait constater à l'assistance que ce sont deux aspects différents du même homme, démontrant par ce fait la complexité de la personnalité en interaction constante. C'est un des thèmes récurrents de l'œuvre littéraire et pédagogique de Medynskij lui-même. Bondarev procède à son autocritique et rappelle ses intentions de départ :

Je veux raconter comment j'ai commencé, quels étaient mes défauts. Avant j'écrivais dans les journaux, j'écrivais des articles. J'ai commencé en 1932. J'ai beaucoup écrit sur la construction du métro. J'écris sur ce que je comprends réellement. [...] Quand j'ai commencé à écrire mon journal, le camarade Medynskij m'a dit que c'était bien mais qu'il n'y avait pas de personnage et que, sans personnage, ce serait mauvais. J'ai commencé à placer un personnage. Cette question m'a demandé beaucoup d'attention. Puisque nous parlons de Kučerenko, il a été mon apprenti, nous étions manœuvres. Maintenant, je suis chef de brigade et lui est un ingénieur important; il a commencé à jouer à l'important...

On peut constater que la difficulté descriptive s'inscrit dans une rivalité relationnelle vécue. Le rapport à l'autre passe par un rapport à soi ; parler de soi en toute authenticité n'est pas facile, d'autant que la discussion est publique. Medynskij généralise l'expérience d'écriture et en tire une définition de l'événement dans le journal, «Le lien entre les événements suit les relations entre les individus et les individus sont montrés dans les événements ${ }^{69}$, ce qu'il résume par la formule « il faut étudier les événements à travers l'homme et l'homme à travers les événements $\gg^{70}$. L'histoire de l'entreprise s'écrit par et à travers les hommes, leurs mains, leurs corps. La fatigue des corps au travail, même transcendée, est lisible dans les entrées des journaux ouvriers.

Le cas de Bondarev est un exemple parmi d'autres du travail pédagogique de Medynskij. Il peut être beaucoup plus sévère lorsqu'il écrit sur le journal de

68. Ibid., d. 239, 1. 143.

69. Ibid., 1. 144.

70. Ibid., d. 416, 1. 32. 
Sapožnikov ${ }^{71}$. Le journal implique la subjectivité de l'auteur, un travail sur luimême qui est exigé de l'auteur ouvrier dans le but d'une perfection de soi. Sapožnikov construit un récit historique et non un journal.

\section{L'art et la méthode du journal ouvrier}

Les clichés et les stéréotypes abondent dans son discours didactique comme dans toutes les productions discursives des années 1930. On trouve dans L'aide méthodique de tenue du journal ouvrier des énoncés injonctifs à la fois thématiques et politiques. Krug voprosov [Ensemble de questions] $]^{72}$ est une réplique des tracts et résolutions de l'Histoire bolchevique des fabriques et des usines ${ }^{73}$. L'aide est rédigée sous la forme d'un guide pour les auteurs de journaux sur la base d'un prédéfini auquel ils sont invités à se conformer. Ces conseils à la fois techniques et idéologiques n'établissent pas de distinction entre la forme et le contenu :

Comment tenir son journal. Les notes du journal doivent être systématiques. Chaque camarade doit avoir un carnet dans lequel il note ses impressions, en deux ou trois mots concernant les événements marquants, pour s'en souvenir. À la maison, ces courtes notes doivent être reportées dans le journal sous une forme déjà plus développée. Les notes quotidiennes doivent être séparées par un intervalle, et devant chacune, il faut indiquer le jour.

71. Ibid, d. 457. Note de Medynskij sur le journal de Sapožnikov le 29 mai 1934,p. 179. « En travaillant sur son journal, le camarade Sapožnikov n'avait pas une conception très claire et précise, d'autant plus que ce travail n'est pas un journal mais l'expérience du puits 29, exprimée sous la forme du journal. Même pour un journal mené parallèlement au travail tel que celui que nous voyons chez Sapožnikov, un tel glissement, une telle superficialité sont incorrects et inadaptés à la forme du journal. L'auteur arrive à des conclusions inacceptables. L'histoire de ce puits est riche en moments très intéressants, des moments clés au cours desquels se sont croisées de nombreuses questions et forces. Ils ne sont pas montrés. Même une question comme l'arrivée du premier millier [de komsomols] est escamotée. Le rôle d'organisation des komsomols est montré en général très faiblement dans le journal. Un problème tel que la lutte pour le puits n'est absolument pas pensé ni montré et il fallait précisément l'éclairer même pour le travail de monteur électricien qui est celui de Sapožnikov. En fonction de ces deux moments cruciaux, on pouvait développer une narration très intéressante, complète et signifiante. Il n'y a rien de tout cela, seulement une approche superficielle, une description peu profonde de faits divers, de petites choses.

Le deuxième défaut qui découle du premier est l'absence d'individus. Les individus sont seulement évoqués mais ne sont en aucune façon dessinés ou caractérisés, ce que l'on aurait pu faire dans la perspective historique qui était celle de Sapožnikov. Et cette absence aggrave les défauts déjà notés chez Sapožnikov. L'absence de réflexion politique claire et précise se ressent dans la langue. Je ne parle pas de ces expressions fautives qui s'expliquent pas le manque d'éducation de l'auteur et qu'un travail avec lui peut rectifier. En définitive, Sapožnikov a abordé le journal comme un exercice littéraire sur la base du mauvais modèle de la mauvaise littérature (literaturščina). De là des effets esthétiques, une attention non critique aux détails, aux rumeurs.

Il est impossible d'imprimer ce journal en l'état. 22 V 1934, T. Medynskij. »

72. GARF, f. 7952, op. 7, d, 231 et 446, 8 pages.

73. Voir « Une fabrique d'écriture », le projet de Gor’kij, dans Pennetier, Pudal, éds., Autobiographies, p. 63-83. 
Série de questions à aborder : le parti, son rôle dirigeant. Les ITR ${ }^{74}$, le rôle de l'ingénieur, le visage du komsomol, la vie internationale, la lutte de classe au métro, la femme au métro, le mode de vie et la culture, tout Moscou construit le métro, toute l'Union construit le métro. [...] Cela ne signifie pas que celui qui tient un journal doive aborder toutes ces questions. Chacun choisit une ou deux de ces questions, selon ses intérêts. [...] L'exigence essentielle du journal intime est la véracité, la sincérité, et l'authenticité. Pas de pseudo-littérature. [...] Écrivez la vérité. Il faut se souvenir que l'essentiel du journal n'est pas la beauté mais la véracité et la totalité des faits.

Sur quoi faut-il attirer l'attention ? Au centre de l'attention [...] doivent être avant tout les gens et les faits. Mais il ne faut pas se limiter aux faits : «Remplissons le plan », «il est survenu un effondrement », « La brigade du Komsomol a décidé de se battre pour le béton komsomol ${ }^{75} »$. Il faut se souvenir que les faits sont l'expression des relations mutuelles entre les gens et, en fin de compte, de la lutte de classes. C'est pourquoi en notant les faits, il faut montrer le rôle qu'y tiennent les individus. Et ces individus ne doivent pas être d'impersonnels «komsomols», «desjatniki», «chefs de brigade». Si tu estimes nécessaire de parler d'une personne, nomme-la [...] En un mot, il faut étudier cet individu sous tous ses aspects, comme une personnalité complexe.

Enfin la dernière chose, dont doit se souvenir tout travailleur qui s'est inclus dans l'Histoire du métro, est que l'histoire ne peut se détacher de notre époque, de la production, de la lutte.

Dans ce texte, le cadre scripturaire est défini par des prescriptions à la fois d'ordre littéraire (le portrait), d'ordre politique (le rôle du parti, du Komsomol) et d'ordre économique (le travail de choc, le rôle des femmes). Les interactions sur les lieux de travail sont désignées comme des signes de la lutte des classes au métro, la lutte entre anciens travailleurs et nouveaux modèles, les travailleurs de choc. L'histoire stalinienne s'inscrit dans les injonctions. Ce qui ne signifie pas pour autant que les ouvriers n'écrivent que ce qui leur est dicté et la pratique de la citation tronquée de journaux dans les puits est largement répandue.

Dans sa théorie générale, Medynskij assigne trois fonctions au journal ouvrier qu'il contextualise dans le genre du journal personnel en Russie :

Bien sûr le journal personnel n'est pas un genre nouveau et le journal ouvrier n'est plus, à notre époque, un cas exceptionnel [...] mais la tenue de masse de journaux et, le plus important, son caractère organisé, font apparaître de façon totalement nouvelle le journal en tant que phénomène littéraire et culturel. [...] Son intérêt historique comme témoignage direct et émanation de la vie; à ce titre, il constitue l'archive de l'histoire, c'est un élément brut qui peut intégrer dans certains cas le récit historique (Histoire du métro de Moscou).

La première fonction consiste à constituer les archives de l'entreprise, une fonction essentielle de témoignage historique. La deuxième est socioculturelle; elle est

74. Inžerno-tehničeskie rabočie, terme désignant les ingénieurs et cadres techniques dans les usines et grands chantiers des années 1930.

75. Production de béton imposée aux équipes de komsomols. 
centrée sur la culture du travail, sur la compétence à s'autoévaluer à travers le travail accompli au service de la collectivité : "savoir regarder avec du recul sa journée de travail et, en relisant les notes, pouvoir faire le bilan du travail accompli ». Enfin, la troisième fonction, qui n'est pas la moins importante aux yeux de Medynskij, est relative au rôle du journal dans la littérature ${ }^{76}$, qu'il devienne un genre autonome ou serve de matériau aux écrivains futurs pour leur roman ${ }^{77}$.

Dans le contexte des années 1930, le journal de production énonce la thématique du journal personnel : se survivre individuellement et collectivement. Le journal s'inscrit dans une double temporalité, comme une lutte contre le temps : il capte le présent de la construction et participe à la construction future du récit historique au moment où l'histoire se fait ; à travers cet acte, le diariste se construit et se survit à lui-même. Il s'opère une capitalisation de l'expérience humaine, une épargne à vie dont les intérêts ne seront peut-être perçus que dans un lointain futur, par les générations à venir.

Dans son argumentaire, Medynskij établit le protocole d'écriture et de lecture de ce qu'il faudrait nommer «le journal personnel de production » avec toutes les ambiguïtés sémantiques de l'expression : le journal est écrit à la première personne et traduit la vision personnelle d'un acte social, l'acte productif résultat d'un travail collectif, voire d'un travail à la chaîne. Les dérapages sont par conséquent inhérents à ce type de pratique. Trop personnels, ils sont anecdotiques et le monde du travail en est absent ; collant trop à la réalité, ils ne se distinguent pas d'autres formes textuelles. D'où les reproches formulés par Medynskij à certains journaux ouvriers - celui de Trušin, par exemple, dont il critique le caractère protocolaire, sec de la relation des faits ${ }^{78}$, le journal se confondant avec le livre de production que chaque brigade rend à la fin de la journée. Le journal peut se définir négativement : ce n'est pas un livre de comptes impersonnel. Il doit se présenter idéalement comme un journal personnel du quotidien de la production, vivant, nourri des rencontres, des figures clés du puits, du rythme de la vie. Les marques énonciatives que critique Medynskij relèvent d'un récit trop bien écrit, vivant, coloré mais anecdotique et auquel fait défaut le contenu idéologique exigé du journal. Medynskij prend deux exemples frappants : «Il n'y avait plus de planches de boisage », écrit Trušin, «Il n'y avait plus de saucisson », répond Hlebnikov ${ }^{79}$. Les difficultés et apories du projet apparaissent dans ces injonctions et conseils. Le diariste doit vivre dans le présent, au jour le jour du journal, dans sa subjectivité fondamentale et, en même temps, découvrir l'essence des faits, établir les liens de causalité, objectiver et comprendre son expérience de travail. Le journal personnel de production est un exercice d'équilibre instable, de tension entre la sphère du privé et du public, du constat et de l'action. Car ce journal d'un ouvrier «conscient » doit répondre au

76. GARF, f. 7952, op. 7, d. 416,1. 15, 16, 17.

77. Sergej P. Antonov, pour son roman Vaska, M. : Molodaja Gvardija, 1988, a utilisé les archives et souvenirs des travailleurs du métro.

78. Ibid., d. 416, 1. 19-22.

79. Ibid., 1. 21. 
« label de la classe ouvrière », écrire et agir, écrire pour mieux produire : «Le véritable homme vivant, auteur ouvrier du journal vit et se bat dans les rangs de sa classe, en prenant part directement à la construction socialiste ${ }^{80}$. C'est ce que Medynskij nomme le contenu idéologique, idejnost', et l'engagement, la prise de parti ou partijnost' du diariste qui, par l'écriture, lutte pour l'élimination des défauts de la production, la construction du métro dans les délais. Le journal est instrumentalisé, arme de la lutte des classes et de l'autovérification, samoproverka. Le diariste ne doit pas seulement noter les problèmes, en prendre conscience par cet acte scripturaire, il doit les résoudre dans le texte et dans la vie : les signaler dans le texte du journal est un premier pas dans la résolution des problèmes de la construction. La dimension pragmatique du journal doit se traduire dans les actes. L'acte de parole est performatif : il agit sur le sujet et le fait agir. Pour Medynskij, la prise de parti ne contredit pas le point de vue personnel du diariste et sa subjectivité (par opposition à la subjectivité petite-bourgeoise, au quant à soi, au ton impersonnel) mais une subjectivité qui s'exerce dans ce schème idéologique. Le problème du «lyrisme» dans le journal découle de cet engagement du scripteur. Medynskij récuse l'enthousiasme de commande, l'emphase rhétorique, les expressions hyperboliques et les passages d'exaltation lyrique « des rythmes de la production » qui sont lus avec ironie. Le lyrisme attendu est celui de la description minutieuse des petites choses de la vie (le réalisme socialiste) : comment, par exemple, Durasova s'est blessée avec sa lampe à kérosène en prenant le thé avant de partir travailler au puits. « La langue du journal doit rester simple et naturelle ${ }^{81}$. Il faut maintenir la bonne distance à l'objet décrit. L'esthétique littéraire de Medynskij lui-même se donne à lire à travers la théorie du journal et sa théorisation de la pratique des auteurs ouvriers, mais cette théorie rend aussi visibles les énoncés contradictoires du réalisme socialiste et des normes linguistiques et idéologiques des années 1930.

Le dernier problème soulevé par Medynskij concerne le destinataire du journal. Celui qui travaille la forme littéraire est dans un rapport de séduction au lecteur. C'est le cas du romancier qui s'adresse à un lecteur, or le diariste ne doit pas écrire dans cet objectif, s'il le fait, il déforme sa pratique ; Medynskij le répète à de nombreuses reprises. Le diariste écrit pour soi, mais paradoxalement, cette écriture est dévoilée et évaluée en public. De plus, le diariste s'inscrit dans un projet collectif précis, celui de l'Histoire des fabriques et des usines. Le journal personnel ou l'œuvre littéraire ne sont pas exclus par Medynskij, ce sont des formes d'expression complémentaires qui ne doivent pas interférer avec le projet de l'Histoire $d u$ métro.

Pendant ces quelques mois de 1934, l'expérience de la pédagogie du journal conduite par Medynskij dans les cercles d'auteurs ouvriers du métro de Moscou témoigne de l'engagement total de son auteur, des difficultés à mener à bien une entreprise périlleuse par ses enjeux et les contraintes idéologiques qui s'exerçaient sur tous les acteurs. Les auteurs ouvriers, peu nombreux, qui se sont réellement

80. Ibid.,1. 23.

81. Ibid., 1.41 
investis dans l'écriture d'un journal y ont découvert pour les plus motivés d'entre eux un plaisir, celui d'écrire, bien éloigné de l'injonction initiale. Les sténogrammes de réunion révèlent un débat, un véritable intérêt pour le journal personnel, de production ou non.

Le cadre institutionnel fut l'une des causes de l'échec du projet : les quelques auteurs ne furent pas retenus pour écrire l'histoire du métro de Moscou, car l'écriture collective de l'histoire à partir des écrits des acteurs était fondamentalement utopique, voire inutile, sinon nuisible, au récit stalinien. D'une part, il était impossible de garantir pour les responsables du parti une orthodoxie, à partir de ce type de pratiques. D'autre part, les journaux collectés étaient décevants, ce dont Medynskij prit conscience en s'adressant à la rédaction :

On a accordé chez nous, déclare-t-il, faisant son autocritique, une très grande importance au journal. Et là, j'ai peut-être eu tort. Quand j'ai commencé à travailler, la mise au point du journal se posait à moi comme une tâche essentielle et généralement nouvelle. Or il s'est trouvé que le mouvement du journal s'est révélé relativement très faible. Il est clair que l'on ne peut se limiter à cela. Le journal n'est qu'un matériau parmi d'autres qui sont complémentaires [...] Le rôle principal doit être joué chez nous par les souvenirs. ${ }^{82}$

Cette expérience scripturaire éclaire l'histoire culturelle des années 1930 à la lumière de ces petites gens qui furent incités à écrire et qui se sont aventurés, pendant quelques semaines pour certains, pendant un an ou plus pour d'autres, sur les sentiers inhabituels de l'écriture de soi. Beaucoup de ces journaux furent ou détruits ou conservés par les diaristes ouvriers qui, comme beaucoup d'autres, tinrent leur journal pendant cette période critique de l'histoire stalinienne.

Phénomène circonscrit aux premières années de l'industrialisation stalinienne, « le journal personnel de production » visait à institutionnaliser le dialogue avec l'autre, à l'adapter au cadre idéologique de l'écriture bolchevique de l'histoire, à placer la sphère privée de l'écriture personnelle au service public de la production, mais aussi à faire émerger l'Homme nouveau à travers la pratique scripturaire. L'apprentissage du journal ouvrier relève d'injonctions contradictoires, celles de l'exhaustivité et de la subjectivité, de l'objectivité et de l'idéologie, d'une vision personnelle et d'un récit politique de l'histoire, plus facile à contrôler par des commissions de spécialistes. La mort de Gor'kij en 1936, la vague d'arrestations de ses proches et de ses collaborateurs de l'Histoire des fabriques et des usines, L. Averbah et A. Kosarev, mirent un terme définitif au projet. La définition du récit s'est jouée aussi dans les relations interpersonnelles des hommes, Kulagin et Medynskij, à la rédaction de l'Histoire du métro : mots d'ordre, application « d'en haut » d'une politique contre la pratique de terrain «d'en bas »; ce conflit idéolo- 
gique de l'époque stalinienne dépasse le cadre de l'Histoire du métro et du projet de Gor'kij d'écrire l'Histoire des fabriques et des usines.

Toutefois, par certains aspects pédagogiques, la pratique organisée de l'écriture de journaux anticipe sur nos ateliers d'écriture. La modernité du projet consiste également dans ces techniques de soi, au sens foucaldien du terme, qui visent dans tout système productif à rendre l'individu plus performant dans son travail et à le faire travailler mieux en le rendant conscient de son travail, en apprenant « à faire le bilan journalier et personnel du travail accompli ${ }^{83}$.

Medynskij sut analyser les fonctions de l'écriture personnelle dans l'entreprise, les transmettre à une minorité ouvrière, qui put s'intégrer et faire carrière dans l'entreprise. Propagandiste et moraliste, la part du pédagogue, écrivain lui-même, influa sur la théorie qu'il en fit. C'est à Medynskij que l'on doit l'effort de généralisation et de théorie à partir de la pratique diaristique. L'engagement personnel de Medynskij, écrivain soviétique ordinaire aujourd'hui oublié, repose sur une pratique régulière du journal, avérée jusqu'à sa dernière publication autobiographique. Il considérait lui-même sa propre vie comme le bilan de soi avec soi, un parcours spirituel jalonné d'étapes cruciales, dans son journal, sa correspondance avec ses lecteurs, comme dans ses œuvres littéraires. Critique et autocritique nourrirent sa réflexion d'écrivain soviétique engagé dans les luttes de son temps en conservant, des années 1920 jusqu'à la fin de sa vie, une sensibilité particulière pour les délinquants et criminels dont il fit le thème de ses œuvres et qu'il chercha à éduquer et à réinsérer dans la société soviétique, s'interrogeant sur la permanence d'un phénomène qui l'inquiétait.

Cercec, EHESS

jbouvard@wanadoo.fr 